\title{
Multi-rogue Wave Solutions for a Generalized Integrable Discrete Nonlinear Schrodinger Equation With Higher-order Excitations
}

Ma Li-Yuan ( $\square$ mly2016@zjut.edu.cn )

Zhejiang University of Technology

Yang Jun

Shanghai Polytechnic University

Zhang Yan-Li

Zhejiang University of Technology

\section{Research Article}

Keywords: Generalized discrete Darboux transformation, Higher-order discrete NLS equation, Higher-order RW

Posted Date: March 26th, 2021

DOI: https://doi.org/10.21203/rs.3.rs-350865/v1

License: (c) (i) This work is licensed under a Creative Commons Attribution 4.0 International License. Read Full License 


\title{
Multi-rogue wave solutions for a generalized integrable discrete nonlinear Schrödinger equation with higher-order excitations
}

\author{
Jun Yang $^{a}$, Yan-Li Zhang ${ }^{b},{\text { Li-Yuan } \mathrm{Ma}^{b} \text {, }}^{*}$ \\ ${ }^{a}$ College of Arts and Sciences, Shanghai Polytechnic University, Shanghai, 201209, P. R. China \\ ${ }^{b}$ Department of Applied Mathematics, Zhejiang University of Technology, Hangzhou 310023, P.R. China
}

\begin{abstract}
In this paper, we construct the discrete rogue wave(RW) solutions for a higher-order or generalized integrable discrete nonlinear Schrödinger(NLS) equation. First, based on the modified Lax pair, the discrete version of generalized Darboux transformation are constructed. Second, the dynamical behaviors of first-, second- and third-order RW solutions are investigated in corresponding to the unique spectral parameter $\lambda$, higher-order term coefficient $\gamma$, and free constants $d_{k}, f_{k}(k=1,2, \cdots, N)$, which exhibit affluent wave structures. The differences between the RW solution of the higher-order discrete NLS equation and that of the Ablowitz-Ladik(AL) equation are illustrated in figures. Moreover, numerical experiments are explored, which demonstrates that strong-interaction RWs are stabler than the weak-interaction RWs. Finally, the modulation instability of continuous waves is studied.
\end{abstract}

Keywords: Generalized discrete Darboux transformation. Higher-order discrete NLS equation. Higher-order RW.

\section{Introduction}

Rogue wave was founded in many fields, such as nonlinear optics, fluid mechanics, and even finance[13]. A mass of nonlinear evolution equations including the NLS equation, Kundu-Eckhaus equation, Hirota equation, Sasa-Satuma equaion and so on, can describe the RW phenomena[4-10]. In discrete integrable system, the RW solutions of the AL equation, coupled discrete NLS equation and discrete Hirota equation are also discussed based on generalized Darboux transformation(DT) and Hirota bilinear method [11-13]. There are great differences on RWs between the continuous integrable system

\footnotetext{
*Corresponding author. Email: mly2016@zjut.edu.cn
} 
and discrete integrable system. Ohta and Yang pointed out that the RWs can exist in the defocusing Ablowitz-Ladik equation [13].

As we know, the higher-order NLS equation named as the Lakshmanan-Porsezian-Daniel(LPD) equation [14]

$$
i q_{t}+q_{x x}+2|q|^{2} q+\gamma\left(q_{x x x x}+8|q|^{2} q_{x x}+6 q^{*} q_{x}^{2}+4 q\left|q_{x}\right|^{2}+2 q^{2} q_{x x}^{*}+6|q|^{4} q\right)=0 .
$$

is a completely integrable fourth order NLS equation. It can describe the dynamics of higher-order alpha-helical proteins with nearest and next nearest neighbour interactions $[15,16]$. This equation has attracted great attentions. In Refs.[14, 17], Authors establish the relation between higher-order NLS equation and one-dimensional Heisenberg ferromagnetic chains when higher order spin-spin exchange interactions (biquadratic type) and the effect of discreteness are considered. The integrability of the Eq.(1) including its singularity structure, construction of Lax pair and Bäcklund transformation etc. have been discussed in detail in Ref.[17]. The one soliton solution of Eq. (1) has been constructed [15] by using Hirota method. Multisoliton solutions using Darboux transformation is presented in [18]. Besides, Eq.(1) can be regarded as a special case for an integrable three-parameter fifth-order nonlinear Schrödinger equation [19, 20]. Rational solutions, breather solutions, rogue wave and modulation instability of this integrable three-parameter fifth-order nonlinear Schrödinger equation are analytically studied based on DT and robust inverse scattering transform [21, 22]. The corresponding rational solutions and breather solutions of Eq.(1) can be obtained under certain constraints.

In this article, we focus on the following spatial discretization [15] of integrable higher-order NLS equation (1)

$$
\begin{aligned}
& i q_{n, t}+\frac{\gamma}{h^{4}}\left(1+\left|q_{n}\right|^{2}\right)\left(\left(1+\left|q_{n-1}\right|^{2}\right) q_{n-2}+\left(1+\left|q_{n+1}\right|^{2}\right) q_{n+2}-4 q_{n-1}-4 q_{n+1}+q_{n}^{*}\left(q_{n+1}^{2}+q_{n-1}^{2}\right)\right. \\
& \left.+q_{n}\left(q_{n-1}^{*} q_{n+1}+q_{n-1} q_{n+1}^{*}\right)+6 q_{n}\right)+\frac{1}{h^{2}}\left(\left(1+\left|q_{n}\right|^{2}\right)\left(q_{n+1}+q_{n-1}\right)-2 q_{n}\right)=0,
\end{aligned}
$$

Eq.(2) can govern the discrete $\alpha$-helical protein chain model with several higher-order excitations and interactions. Under the transformation

$$
q_{n}(t)=h q(n h, t) \triangleq h q(x, t),
$$

the higher-order integrable discrete NLS equation (2) yields the integrable fourth-order NLS equation (1). Ref.[15] investigates the integrability of eq.(2) including Hamiltonian, discrete Lax pair, discrete soliton and gauge equivalence. However, as we know, there is little work on rogue wave solutions and breather solutions of this higher-order integrable discrete NLS equation (2). This is the main motivation for us to investigate the higher-order RWs of the discrete integrable NLS equation (2) with higher-order excitations in this paper. Moreover, it is very meaningful to study other integrable 
properties of the higher-order integrable discrete NLS equation (2). We shall give an insight into the continuous limit theory of higher-order integrable discrete NLS equation (2) including discrete DT, discrete rational solutions, discrete breather solutions and gauge equivalence in the future. The paper is organized as follows. In Sect.2, by using the modified discrete Lax pairs, we apply the generalized $(1, \mathrm{~N}-1)$-fold Darboux transformation [4, 9] to construct higher-order discrete RW solutions of Eq.(2). The dynamical behaviors of these discrete RWs are discussed in Sect.3, which exhibits interesting wave structures. Finally, in Sect.4 the modulation instability of continuous-wave states of the higher-order discrete NLS equation (2) is investigated.

\section{Lax pair and generalized discrete DT}

The higher-order discrete NLS equation(2) admits the following discrete modified Lax pair

$$
E \varphi_{n}=U_{n} \varphi_{n}, \quad \varphi_{n, t}=V_{n} \varphi_{n}
$$

where the shift operator $E$ is defined as $E \varphi_{n}=\varphi_{n+1}$, the vector eigenfunction $\varphi_{n}=\left(\varphi_{n, 1}, \varphi_{n, 2}\right)^{T}$ and the matrices $U_{n}$ and $V_{n}$ take the forms

$$
\begin{aligned}
U_{n} & =\left(\begin{array}{cc}
\lambda & q_{n} \lambda^{-1} \\
-q_{n}^{*} \lambda & \lambda^{-1}
\end{array}\right), \\
V_{n} & =\frac{i \gamma}{h^{4}}\left(\begin{array}{cc}
A_{n}\left(\lambda, \lambda^{-1}, q_{n}\right) & B_{n}\left(\lambda, \lambda^{-1}, q_{n}\right) \\
B_{n}\left(\lambda^{-1}, \lambda, q_{n}^{*}\right) & -A_{n}\left(\lambda^{-1}, \lambda, q_{n}^{*}\right)
\end{array}\right)+\frac{i}{h^{2}}\left(\begin{array}{cc}
C_{n}\left(\lambda, \lambda^{-1}, q_{n}\right) & D_{n}\left(\lambda, \lambda^{-1}, q_{n}\right) \\
D_{n}\left(\lambda^{-1}, \lambda, q_{n}^{*}\right) & -C_{n}\left(\lambda^{-1}, \lambda, q_{n}^{*}\right)
\end{array}\right),
\end{aligned}
$$

with

$$
\begin{aligned}
A_{n}\left(\lambda, \lambda^{-1}, q_{n}\right)= & \frac{\lambda^{4}+\lambda^{-4}}{2}+\lambda^{2}\left(q_{n} q_{n-1}^{*}-2\right)+\lambda^{-2}\left(q_{n}^{*} q_{n-1}-2\right)-4 q_{n} q_{n-1}^{*} \\
& +q_{n}^{2} q_{n-1}^{* 2}+\left(1+\left|q_{n-1}\right|^{2}\right) q_{n} q_{n-2}^{*}+\left(1+\left|q_{n}\right|^{2}\right) q_{n+1} q_{n-1}^{*}+3, \\
B_{n}\left(\lambda, \lambda^{-1}, q_{n}\right)= & \lambda^{2} q_{n}-\lambda^{-4} q_{n-1}+\left(1+\left|q_{n}\right|^{2}\right) q_{n+1}+q_{n}^{2} q_{n-1}^{*}-4 q_{n} \\
& -\lambda^{-2}\left(\left(1+\left|q_{n-1}\right|^{2}\right) q_{n-2}+q_{n}^{*} q_{n-1}^{2}-4 q_{n-1}\right), \\
C_{n}\left(\lambda, \lambda^{-1}, q_{n}\right)= & \lambda^{2}-1-\left(\lambda-\lambda^{-1}\right)+q_{n} q_{n-1}^{*}, \\
D_{n}\left(\lambda, \lambda^{-1}, q_{n}\right)= & q_{n}-q_{n-1} \lambda^{-2} .
\end{aligned}
$$

One can directly verify that the discrete zero curvature condition $U_{n, t}=\left(E V_{n}\right) U_{n}-U_{n} V_{n}$ of the linear spectral equations (4) yields the generalized integrable discrete NLS equation (2).

Following the idea in [23], the Darboux transformation of the higher-order discrete NLS equation(2) can be obtained. Under the gauge transformation

$$
\psi_{n}^{[1]}=T_{n}[N](\lambda) \psi_{n},
$$


with

$$
T_{n}[N]=\left(\begin{array}{cc}
\lambda^{N}+\sum_{k=1}^{N} T_{n, 1}^{(N-2 k)} \lambda^{N-2 k} & \sum_{k=1}^{N} T_{n, 2}^{(N-2 k+1)} \lambda^{N-2 k} \\
(-1)^{N+1} \sum_{k=1}^{N} T_{n, 2}^{(N-2 k+1) *} \lambda^{-N+2 k} & (-1)^{N}\left(\lambda^{-N}+\sum_{k=1}^{N} T_{n, 1}^{(N-2 k) *} \lambda^{-N+2 k}\right)
\end{array}\right),
$$

where $T_{n, 1}^{(N-2 k)}$ and $T_{n, 2}^{(N-2 k+1)}$ can be determined by

$$
\begin{aligned}
& \left(\lambda_{j}^{N}+\sum_{k=1}^{N} T_{n, 1}^{(N-2 k)} \lambda_{j}^{N-2 k}\right) \varphi_{n, 1}^{(j)}+\left(\sum_{k=1}^{N} T_{n, 2}^{(N-2 k+1)} \lambda^{N-2 k}\right) \varphi_{n, 2}^{(j)}=0, \\
& \left(\left(\lambda_{j}^{*}\right)^{-N}+\sum_{k=1}^{N} T_{n, 1}^{(N-2 k)}\left(\lambda_{j}^{*}\right)^{-N+2 k}\right) \varphi_{n, 2}^{(j) *}-\left(\sum_{k=1}^{N} T_{n, 2}^{(N-2 k+1)}\left(\lambda_{j}^{*}\right)^{-N+2 k}\right) \varphi_{n, 1}^{(j) *}=0 .
\end{aligned}
$$

The linear spectral problem(4) changes to new one as

$$
E \tilde{\psi}_{n}=\tilde{U}_{n} \psi_{n}, \quad \frac{d \tilde{\psi}_{n}}{d t}=\tilde{V}_{n} \psi_{n}
$$

and the matrices $\tilde{U}_{n}$ and $\tilde{U}_{n}$ satisfy

$$
\tilde{U}_{n}=T_{n+1}[N] U_{n}\left(T_{n}[N]\right)^{-1}, \quad \tilde{U}_{n}=\left(T_{n, t}[N]+T_{n}[N] V_{n}\right)\left(T_{n}[N]\right)^{-1},
$$

The relation between potential $\tilde{q}_{n}[N]$ and potential $q_{n}$ is

$$
\tilde{q}_{n}[N]=-q_{n} T_{n+1,1}^{(-N)}-T_{n+1,2}^{(-N+1)}
$$

where

$$
T_{n, 1}^{(-N)}=-\frac{\Omega_{1}[N]}{\Omega[N]}, \quad T_{n, 2}^{(-N+1)}=-\frac{\Omega_{2}[N]}{\Omega[N]},
$$

with

$$
\Omega[N]=\left|\begin{array}{ccccccc}
\lambda_{1}^{-N} \varphi_{n, 1}^{(1)} & \lambda_{1}^{-N} \varphi_{n, 2}^{(1)} & \lambda_{1}^{-N+2} \varphi_{n, 1}^{(1)} & \lambda_{1}^{-N+2} \varphi_{n, 2}^{(1)} & \cdots & \lambda_{1}^{N-2} \varphi_{n, 1}^{(1)} & \lambda_{1}^{N-2} \varphi_{n, 2}^{(1)} \\
\lambda_{2}^{-N} \varphi_{n, 1}^{(2)} & \lambda_{2}^{-N} \varphi_{n, 2}^{(2)} & \lambda_{2}^{-N+2} \varphi_{n, 1}^{(2)} & \lambda_{2}^{-N+2} \varphi_{n, 2}^{(2)} & \cdots & \lambda_{2}^{N-2} \varphi_{n, 1}^{(2)} & \lambda_{2}^{N-2} \varphi_{n, 2}^{(2)} \\
\vdots & \vdots & \vdots & \vdots & \vdots & \vdots & \vdots \\
\lambda_{N}^{-N} \varphi_{n, 1}^{(N)} & \lambda_{N}^{-N} \varphi_{n, 2}^{(N)} & \lambda_{N}^{-N+2} \varphi_{n, 1}^{(N)} & \lambda_{N}^{-N+2} \varphi_{n, 2}^{(N)} & \cdots & \lambda_{N}^{N-2} \varphi_{n, 1}^{(N)} & \lambda_{N}^{N-2} \varphi_{n, 2}^{(N)} \\
\left(\lambda_{1}^{*}\right)^{N} \varphi_{n, 2}^{(1) *} & -\left(\lambda_{1}^{*}\right)^{N} \varphi_{n, 1}^{(1) *} & \left(\lambda_{1}^{*}\right)^{N-2} \varphi_{n, 2}^{(1) *} & -\left(\lambda_{1}^{*}\right)^{N-2} \varphi_{n, 1}^{(1) *} & \cdots & \left(\lambda_{1}^{*}\right)^{-N+2} \varphi_{n, 2}^{(1) *} & -\left(\lambda_{1}^{*}\right)^{-N+2} \varphi_{n, 1}^{(1) *} \\
\left(\lambda_{2}^{*}\right)^{N} \varphi_{n, 2}^{(2) *} & -\left(\lambda_{2}^{*}\right)^{N} \varphi_{n, 1}^{(2) *} & \left(\lambda_{2}^{*}\right)^{N-2} \varphi_{n, 2}^{(2) *} & -\left(\lambda_{2}^{*}\right)^{N-2} \varphi_{n, 1}^{(2) *} & \cdots & \left(\lambda_{2}^{*}\right)^{-N+2} \varphi_{n, 2}^{(2) *} & -\left(\lambda_{2}^{*}\right)^{-N+2} \varphi_{n, 1}^{(2) *} \\
\vdots & \vdots & \vdots & \vdots & \vdots & \vdots & \vdots \\
\left(\lambda_{N}^{*}\right)^{N} \varphi_{n, 2}^{(N) *} & -\left(\lambda_{N}^{*}\right)^{N} \varphi_{n, 1}^{(N) *} & \left(\lambda_{N}^{*}\right)^{N-2} \varphi_{n, 2}^{(N) *} & -\left(\lambda_{N}^{*}\right)^{N-2} \varphi_{n, 1}^{(N) *} & \cdots & \left(\lambda_{N}^{*}\right)^{-N+2} \varphi_{n, 2}^{(N) *} & -\left(\lambda_{N}^{*}\right)^{-N+2} \varphi_{n, 1}^{(N) *}
\end{array}\right|
$$

It is noted that the expression of $\Omega_{1}[N]$ and $\Omega_{2}[N]$ can be derived by substituting $\left(\lambda_{1}^{N} \varphi_{n, 1}^{(1)}, \lambda_{2}^{N} \varphi_{n, 1}^{(2)}, \cdots\right.$ $\left.\cdot, \lambda_{N}^{N} \varphi_{n, 1}^{(N)},\left(\lambda_{1}^{*}\right)^{-N} \varphi_{n, 2}^{(1) *},\left(\lambda_{2}^{*}\right)^{-N} \varphi_{n, 2}^{(2) *}, \cdots,\left(\lambda_{N}^{*}\right)^{-N} \varphi_{n, 2}^{(N) *}\right)^{\mathrm{T}}$ for the first and second column in $\Omega[N]$, respectively. 
Next, we will construct the generalized $(1, N-1)$-fold DT for higher-order discrete NLS equation(2). The generalized $(1, N-1)$-fold DT links to single spectral parameter $\lambda=\lambda_{1}$ and the order N-1 of the highest-order derivatives for the eigenfunctions. Using the similar method in Ref.[9, 12], we get a generalized (1, $N-1$ )-fold DT for higher-order discrete NLS equation(2). Especially, we consider the following eigenfunction solution of the Lax pair(4) with seed solution $q_{0}(n, t)=c e^{i \phi t}$

$$
\varphi(\lambda)=\left(\begin{array}{c}
\left(C_{1} \chi_{1}^{-} e^{n \ln \nu_{1}^{-}+\mu_{1}^{-} t}+C_{2} \chi_{1}^{+} e^{n \ln \nu_{1}^{+}+\mu_{1}^{+} t}\right) e^{i \phi t+\Phi\left(\epsilon^{2}\right)} \\
\left(C_{1} e^{n \ln \nu_{1}^{-}+\mu_{1}^{-} t}+C_{2} e^{n \ln \nu_{1}^{+}+\mu_{1}^{+} t}\right) e^{-i \phi t+\Phi\left(\epsilon^{2}\right)}
\end{array}\right),
$$

where

$$
\begin{aligned}
\phi & =\frac{2 c^{2}\left(3 c^{2} \gamma+h^{2}\right)}{h^{4}}, \Phi\left(\epsilon^{2}\right)=\sqrt{\left(\lambda^{2}-1\right)-4 \lambda^{2} c^{2}} \sum_{k=1}^{N}\left(d_{k}+i f_{k}\right) \epsilon^{2 k} \\
\chi_{1}^{ \pm} & =\frac{1-\lambda^{2} \mp \sqrt{\lambda^{4}-2\left(1+2 c^{2}\right) \lambda^{2}+1}}{2 c \lambda^{2}}, \nu_{1}^{ \pm}=\lambda+\frac{c}{\lambda \chi_{1}^{ \pm}}, \\
\mu_{1}^{ \pm} & =\frac{i\left(\lambda^{2}-1\right)\left(h^{2} \lambda^{2}(\lambda-1)^{2} \pm\left(\gamma \lambda^{4}+\left(h^{2}+2\left(c^{2}-1\right) \gamma\right) \lambda^{2}+\gamma\right) \sqrt{\lambda^{4}-2\left(2 c^{2}+1\right) \lambda^{2}+1}\right)}{2 h^{4} \lambda^{4}}
\end{aligned}
$$

with $C_{j}(j=1,2)$ are arbitrary complex parameters(i.e., $\left.C_{1}=1, C_{2}=0\right), d_{k}, f_{k}$ are free real and $\epsilon$ is small parameter. We fix the spectral parameter $\lambda=\lambda_{1}+\epsilon^{2}$ with $\lambda_{1}=\sqrt{1+c^{2}} \pm c$ in Eq.(11) and expand eigenfunction $\varphi(\lambda)$ into the Taylor series at $\epsilon=0$. We obtain

$$
\begin{aligned}
& \left(\lambda_{1}+\epsilon^{2}\right)^{j} \phi_{1}\left(\lambda_{1}+\epsilon^{2}\right)=\lambda_{1}^{j} \phi_{1}+\phi_{1}[j, 1] \epsilon^{2}+\cdots+\phi_{1}[j, N] \epsilon^{2 N}+\cdots, \\
& \left(\lambda_{1}+\epsilon^{2}\right)^{j} \psi_{1}\left(\lambda_{1}+\epsilon^{2}\right)=\lambda_{1}^{j} \psi_{1}+\psi_{1}[j, 1] \epsilon^{2}+\cdots+\psi_{1}[j, N] \epsilon^{2 N}+\cdots,
\end{aligned}
$$

where

$$
\begin{aligned}
\phi_{1}[j, l] & =\left.\frac{1}{(2 l) !} \frac{\partial^{2 l}}{\partial \epsilon^{2 l}}\left[\left(\lambda_{1}+\epsilon^{2}\right)^{j} \phi_{1}\left(\lambda_{1}+\epsilon^{2}\right)\right]\right|_{\epsilon=0}, \\
\psi_{1}[j, l] & =\left.\frac{1}{(2 l) !} \frac{\partial^{2 l}}{\partial \epsilon^{2 l}}\left[\left(\lambda_{1}+\epsilon^{2}\right)^{j} \psi_{1}\left(\lambda_{1}+\epsilon^{2}\right)\right]\right|_{\epsilon=0} . \\
j & =-N,-N+1, \cdots, 0,1, \cdots, N-1, N ; l=1,2, \cdots
\end{aligned}
$$

Then we obtain a generalized DT for the higher-order discrete NLS equation(2)

$$
q_{n}[N]=-q_{0} f_{n+1}^{(-N)}-g_{n+1}^{(-N+1)},
$$

where

$$
f_{n}^{(-N)}=-\frac{\triangle_{1}^{[N]}}{\triangle^{[N]}}, \quad g_{n}^{(-N+1)}=-\frac{\triangle_{2}^{[N]}}{\triangle^{[N]}},
$$


with

$$
\triangle^{[N]}=\left|\begin{array}{cccccccc}
\lambda^{-N-1} \phi_{1} & \phi_{1}[-N-1,1] & \ldots & \phi_{1}[-N-1, N] & \lambda^{* N+1} \psi_{1}^{*} & \psi_{1}[N+1,1]^{*} & \ldots & \psi_{1}[N+1, N]^{*} \\
\lambda^{-N-1} \psi_{1} & \psi_{1}[-N-1,1] & \cdots & \psi_{1}[-N-1, N] & -\lambda^{* N+1} \phi_{1}^{*} & -\phi_{1}[N+1,1]^{*} & \ldots & -\phi_{1}[N+1, N]^{*} \\
\lambda^{-N+1} \phi_{1} & \phi_{1}[-N+1,1] & \cdots & \phi_{1}[-N+1, N] & \lambda^{* N-1} \psi_{1}^{*} & \psi_{1}[N-1,1]^{*} & \ldots & \psi_{1}[N-1, N]^{*} \\
\lambda^{-N+1} \psi_{1} & \psi_{1}[-N+1,1] & \cdots & \psi_{1}[-N+1, N] & -\lambda^{* N-1} \phi_{1}^{*} & -\phi_{1}[N-1,1]^{*} & \ldots & -\phi_{1}[N-1, N]^{*} \\
\vdots & \vdots & \ddots & \vdots & \vdots & \vdots & \ddots & \vdots \\
\lambda^{N-1} \phi_{1} & \phi_{1}[N-1,1] & \ldots & \phi_{1}[N-1, N] & \lambda^{*-N+1} \psi_{1}^{*} & \psi_{1}[-N+1,1]^{*} & \ldots & \psi_{1}[-N+1, N]^{*} \\
\lambda^{N-1} \psi_{1} & \psi_{1}[N-1,1] & \cdots & \psi_{1}[N-1, N] & -\lambda^{*-N+1} \phi_{1}^{*} & -\phi_{1}[-N+1,1]^{*} & \cdots & -\phi_{1}[-N+1, N]^{*}
\end{array}\right|
$$

The matrix $\triangle_{1}^{[N]}$ and $\triangle_{2}^{[N]}$ are described by $\triangle^{[N]}$ respectively, but the first row and the second row in the $\triangle^{[N]}$ are changed to $\left(\lambda^{N+1} \phi_{1}, \cdots, \phi_{1}[N+1, N], \lambda^{*-N-1} \psi_{1}^{*}, \cdots, \psi_{1}[-N-1, N]^{*}\right)$, respectively.

\section{RW solutions and dynamic behaviors}

\section{Case 1: one-order RW solutions}

As $N=1$, the solution (13) reduces

$$
q_{n}[1]=-q_{0} f_{n+1}^{(-1)}-g_{n+1}^{(0)}
$$

with

$$
\Delta^{[1]}=\left|\begin{array}{cccc}
\lambda^{-2} \phi_{1} & \phi_{1}[-2,1] & \lambda^{* 2} \psi_{1}^{*} & \psi_{1}[2,1]^{*} \\
\lambda^{-2} \psi_{1} & \psi_{1}[-2,1] & -\lambda^{* 2} \phi_{1}^{*} & -\phi_{1}[2,1]^{*} \\
\phi_{1} & \phi_{1}[0,1] & \psi_{1}^{*} & \psi_{1}[0,1]^{*} \\
\psi_{1} & \psi_{1}[0,1] & -\phi_{1}^{*} & -\phi_{1}[0,1]^{*}
\end{array}\right| .
$$

For convenient, choose $h=1, c=\frac{3}{4}$ corresponding to $\lambda_{1}=\frac{1}{2}$ then we obtain the first-order RW solution of Eq.(2)

$$
q_{n}[1]=-\frac{3}{4} e^{\frac{9}{128} i(16+27 \gamma) t} \frac{12288 n(3 n-2)+225 t(8+27 \gamma)(9 t(8+27 \gamma)-128 i)-81920}{12288 n(3 n-2)+5\left(4096+405 t^{2}(8+27 \gamma)^{2}\right)},
$$

Note that $q_{n+n_{0}}[1]$ is also a solution with arbitrary real number $n_{0}$ and the translation equally meets the higher-order RW solutions. We illustrate the property of first-order RW solution (15).

By analyzing the explicit formula of $q_{n+n_{0}}[1]$, we find that the parameter $\gamma$ produces no effect in the amplitude of the first-order RW solution (15). The maximum amplitude of $\left|q_{\mathrm{n}, \max }[1]\right|$ is $\frac{63}{16}$ at point $\left(n_{1}, t_{1}\right)=(0,0)$ with the shift $n_{0}=\frac{1}{3}$, which is an on-site RW(see Fig.1(a)). The minima amplitude attains 0 at two sites $\left(n_{2}, t_{2}\right)=(-1,0),\left(n_{3}, t_{3}\right)=(1,0)$ with the shift $n_{0}=\frac{4-\sqrt{21}}{3}, \frac{\sqrt{21}-2}{3}$ respectively. Moreover, we find that the lower peak amplitude of the first-order RW can reach at two adjacent lattice sites when $n_{0}=\frac{5}{6}$, which is called inter-site RW(see Fig.1(b)). Compared with the fundamental RW solutons in the AL equation[12], we can see that the higher-order discrete NLS equation(2) has the same extremum amplitude but different center points. 


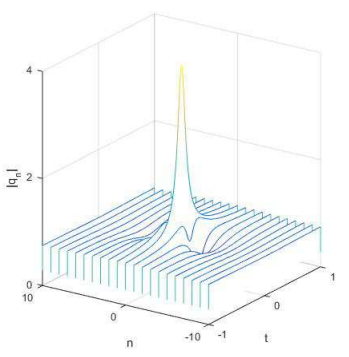

(a)

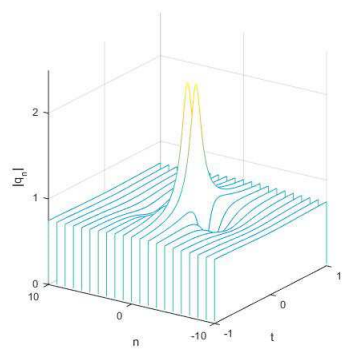

(b)

Figure 1: First-order RW solution (15) with $\gamma=1$ : (a) on-site $\operatorname{RW}\left(n_{0}=1 / 3\right)$; (b) inter-site $\operatorname{RW}\left(n_{0}=5 / 6\right)$.

Next, we consider the effect of higher-order term parameter $\gamma$ and $\lambda$ on RWs. Figures 2(a) and 2(c) show that the first-order RW become narrower with the increase of nonlinear term parameter $\gamma$ but the peak does not changed. When $\gamma \rightarrow \infty$, the first-order RWs become narrower and narrower; On the conversely, when $\gamma \rightarrow 0$ the higher-order discrete NLS equation(2) reduces to the AL equation, and RWs approach the fundamental RWs of the AL equation. Altering the parameter $\lambda$, we see that the amplitude of the fist-order rogue wave increase with the spectrum $\lambda$ increase(see Figs.2(d) and $2(\mathrm{e}))$.

In what follows, we study the dynamical behaviors of the first-order RW solutions by numerical simulation with the initial conditions and perturbation for the Eq.(2). Fig. 3(a) is the exact first-order RW solution(15). Figures 3(b)and 3(c) are the profiles of the numerical simulation, which exhibit the time evolution of the RW with initial condition and the perturbation with $2 \%$ amplitude as random noise at $t \in(-1,1)$. The corresponding results show that the numerical simulations of the first-order RW solution can well agree with the exact RW solution(15) and the perturbation case only has a little weak oscillation near the edges.

Case 2: second-order RW solutions

Formula (13) with $N=2$ yields

$$
q_{n}[2]=-q_{0} f_{n+1}^{(-2)}-g_{n+1}^{(-1)}
$$

where

$$
f_{n}^{(-2)}=-\frac{D_{1}^{[2]}}{D^{[2]}}, \quad g_{n}^{(-1)}=-\frac{D_{2}^{[2]}}{D^{[2]}}
$$




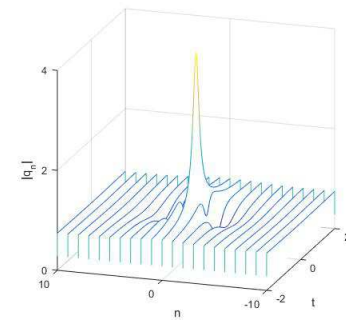

(a)

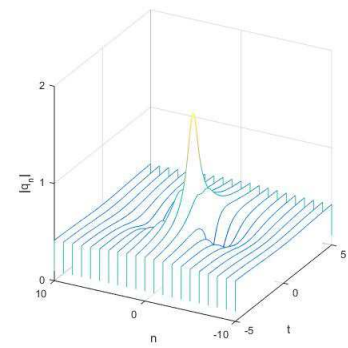

(d)

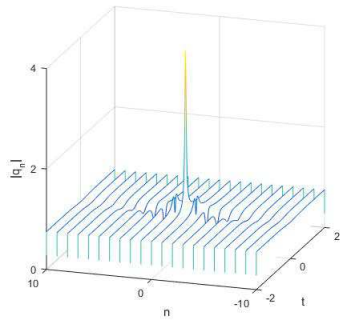

(b)

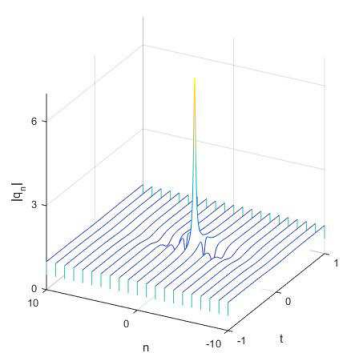

(e)

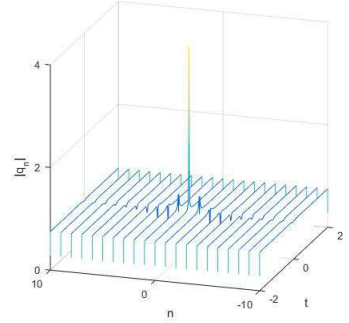

(c)

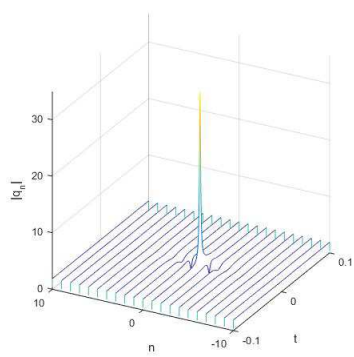

(f)

Figure 2: First-order RWs (15) with $n_{0}=1 / 3$ and the different parameter $\gamma:(\mathrm{a}) \gamma=1 / 5 ;(\mathrm{b}) \gamma=2 ;$ (c) $\gamma=10$ and plots with different parameter $\lambda$ :(d) $\lambda=2 / 3$, the shift $n_{0}=4 / 5$, (e) $\lambda=1+\sqrt{2}$, the shift $n_{0}=(1-$ $\sqrt{2}) / 2,(\mathrm{f}) \lambda=4$, the shift $n_{0}=-1 / 15$.

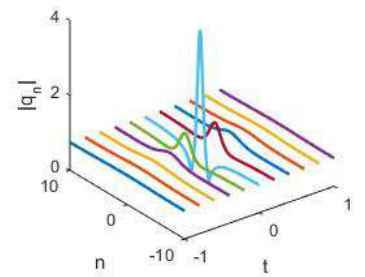

(a)

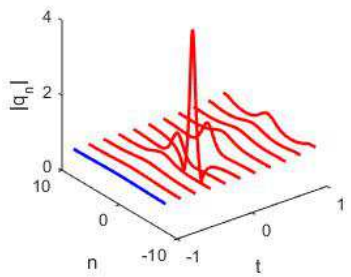

(b)

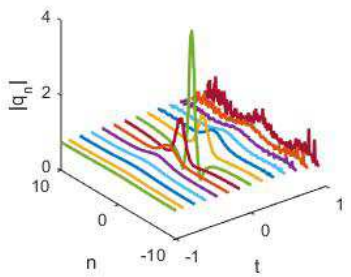

(c)

Figure 3: The first-order RW solutions(15). (a) Exact solution, (b) numerical simulations using exact solutions(15) with $t=-1$. (c) numerical simulations by adding random noise with amplitude $2 \%$ as the initial condition. 
with

$$
\Delta^{[2]}=\left|\begin{array}{cccccc}
\lambda^{-3} \phi_{1} & \phi_{1}[-3,1] & \phi_{1}[-3,2] & \lambda^{* 3} \psi_{1}^{*} & \psi_{1}[3,1]^{*} & \psi_{1}[3,2]^{*} \\
\lambda^{-3} \psi_{1} & \psi_{1}[-3,1] & \psi_{1}[-3,2] & -\lambda^{* 3} \phi_{1}^{*} & -\phi_{1}[3,1]^{*} & -\phi_{1}[3,2]^{*} \\
\lambda^{-1} \phi_{1} & \phi_{1}[-1,1] & \phi_{1}[-1,2] & \lambda^{*} \psi_{1}^{*} & \psi_{1}[1,1]^{*} & \psi_{1}[1,2]^{*} \\
\lambda^{-1} \psi_{-1} & \psi_{1}[-1,1] & \psi_{1}[-1,2] & -\lambda^{*} \phi_{1}^{*} & -\phi_{1}[1,1]^{*} & -\phi_{1}[1,2]^{*} \\
\lambda \phi_{1} & \phi_{1}[1,1] & \phi_{1}[1,2] & \lambda^{*-1} \psi_{1}^{*} & \psi_{1}[-1,1]^{*} & \psi_{1}[-1,2]^{*} \\
\lambda \psi_{1} & \psi_{1}[1,1] & \psi_{1}[1,2] & -\lambda^{*-1} \phi_{1}^{*} & -\phi_{1}[-1,1]^{*} & -\phi_{1}[-1,2]^{*}
\end{array}\right|,
$$

and $\Delta_{1}^{[2]}$ and $\Delta_{2}^{[2]}$ are described by $\Delta^{[2]}$, but the first row and the second row are replace by $\left(\lambda_{1}^{3} \phi_{1}, \phi_{1}[3,1], \phi_{1}[3,2], \lambda_{1}^{*-3} \psi_{1}^{*}, \psi_{1}[-3,1]^{*}, \psi_{1}[-3,2]^{*}\right)$ in the $\Delta^{[2]}$, respectively.

If we choose $c=3 / 4, \lambda=2, h=\gamma=1$, the exact second-order RW solutions can be expressed as

$$
q_{n}[2]=-\left.\frac{3}{4} e^{\frac{387}{128} i t} \frac{A_{2}(n, t)}{B_{2}(n, t)}\right|_{n \rightarrow n+n_{0}}
$$

where $B_{2}(n, t), A_{2}(n, t)$ are obtained through mathematica software:

$$
\begin{aligned}
B_{2}(n, t)= & 68719476736(1600+3 n(1280+3 n(497+3 n(136+3 n(26+n(8+n)))))+849346560000(23465 \\
+ & 21 n(-2056+3 n(-96+7 n(16+3 n)))) t^{2}+1200225600000000(670+21 n(8+3 n)) t^{4} \\
+ & 1696058545166015625 t^{6}+1061683200\left(25 t\left(4096(-125+21 n(8+3 n))-5788125 t^{2}\right) d_{1}\right. \\
+ & \left.3276800 d_{1}^{2}+64 e_{1}\left(4096 n(1+n)(3+n)-275625(4+3 n) t^{2}+51200 e_{1}\right)\right), \\
A_{2}(n, t)= & 68719476736(100+3 n(-1520+3 n(-428+n(8+3 n(53+3 n(8+n))))))-2415919104000 i(-4880 \\
& +3 n(-1336+3 n(-6+7 n(16+3 n)))) t+2548039680000(-29220+7 n(-4856+3 n(-446 \\
& +7 n(16+3 n)))) t^{2}-975421440000000 i(275+21 n(8+3 n)) t^{3}+1200225600000000(-205 \\
& +21 n(8+3 n)) t^{4}-2067576131250000000 i t^{5}+1696058545166015625 t^{6}+589824000( \\
& \left.-262144 i(20+3 n(8+3 n))+184320(50+21 n(8+3 n)) t+158760000 i t^{2}-260465625 t^{3}\right) d_{1} \\
& +3478923509760000 d_{1}^{2}+7549747200 e_{1}(4096(100+3 n(34+3 n(4+n))) \\
& \left.+1008000 i(4+3 n) t-2480625(4+3 n) t^{2}+460800 e_{1}\right) .
\end{aligned}
$$

We see that the parameters $d_{1}$ and $f_{1}$ control the strong and weak interaction of the second-order RW(17).

- For the case $d_{1}=f_{1}=0$, the strong interaction happens that the RWs have four minimum points and five local maximum including a biggest peak at the center of the wave packets(see Fig.4(a)).

- For the case $d_{1} \neq 0$ or $f_{1} \neq 0$, the second-order RWs split into three first-order RWs, whose centers become a rotating triangle, and the whole profiles have three local maximum and six minimum points(see Figs.4(b) and 4(c)). 


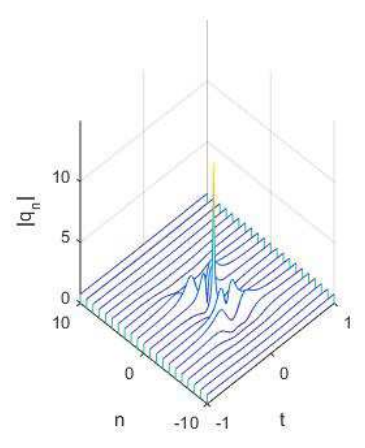

(a)

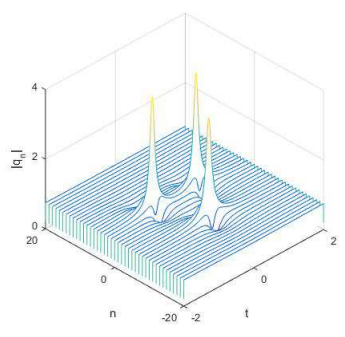

(b)

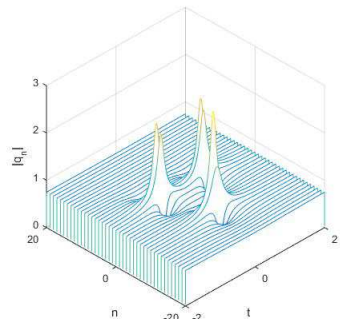

(c)

Figure 4: The second-order discrete RW solutions (17) with $c=3 / 4, \lambda=2, h=\gamma=1$. (a) $d_{1}=f_{1}=0$, $n_{0}=0 ;$ (b) $d_{1}=10, f_{1}=0, n_{0}=-5 / 6 ;$ (c) $d_{1}=0, f_{1}=10, n_{0}=2 / 3$.

Moreover, adjusting the parameters freely, we find that the area of the triangle increases with the increase of the parameters $\left|d_{1}\right|$ or $\left|f_{1}\right|$ and $\left|f_{1}\right|$ can control the rotation of the triangle RWs.

Next, we give the dynamical property for the second-order RWs by the numerical simulation. Figures $5(\mathrm{a})$ and $5(\mathrm{~d})$ are exact second-order RW solutions with different parameters $d_{1}$ and $e_{1}$. Figures 5(b) and 5(c) show that the numerical simulation of the second-order RW solutions well agree with the exact solution except for weak oscillations at $t>0.4$ (see Figs. 5(b) and 5(e)). If we add the random noise $(2 \%)$ to the initial solution, the strong interaction case (i.e., $d_{1}=0, f_{1}=0$ ) plays with great consistency(see Fig. $5(\mathrm{c})$ ) but the weak interaction case (i.e., $d_{1}=10, f_{1}=0$ ) shows severely oscillations after time exceeds 0.2 , which may be due to the main energy distribution[12].

\section{Case 3: third-order RW solutions}

When $N=3$, by the formula (13) and take the special spectral parameters $\lambda=\frac{7}{4}$ with $c=\frac{33}{56}$, then the third-order discrete RW solution is obtained as

$$
q_{n}[3]=-q_{0} f_{n+1}^{(-3)}-g_{n+1}^{(-2)}
$$

where

$$
f_{n}^{(-3)}=-\frac{\Delta_{1}^{[3]}}{\Delta^{[3]}}, \quad g_{n}^{(-2)}=-\frac{\Delta_{2}^{[3]}}{\Delta^{[3]}},
$$




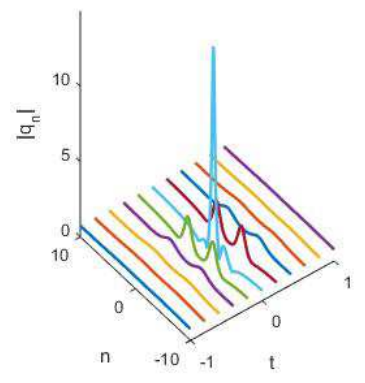

(a)

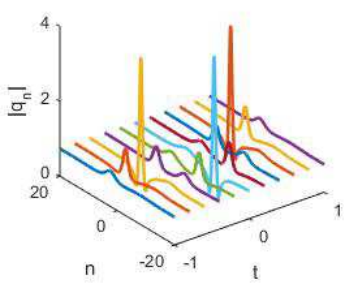

(d)

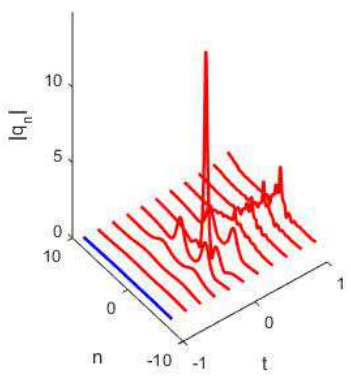

(b)

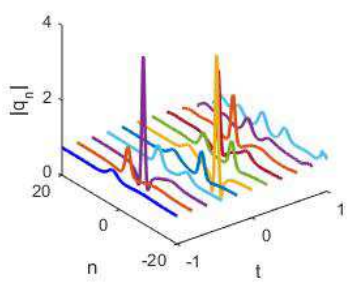

(e)

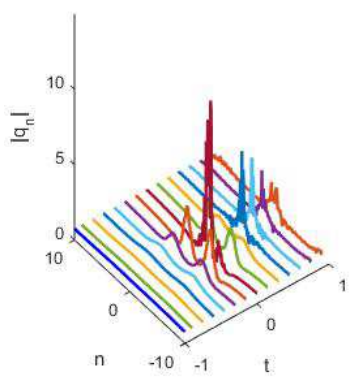

(c)

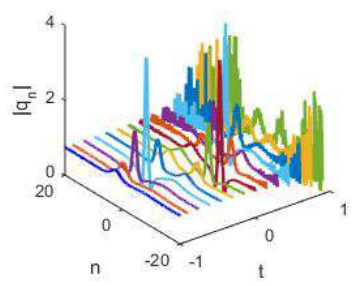

(f)

Figure 5: The second-order RW solutions(17). Exact solutions with (a) $d_{1}=f_{1}=0$ and (d) $d_{1}=10,=f_{1}=$ 0 . (b) and (e) the numerical simulation using exact solutions(17) at $t=-1$ as initial conditions. (d) and (f) numerical simulations by adding random noise with amplitude $2 \%$ to the exact solutions(17) as initial conditions. 


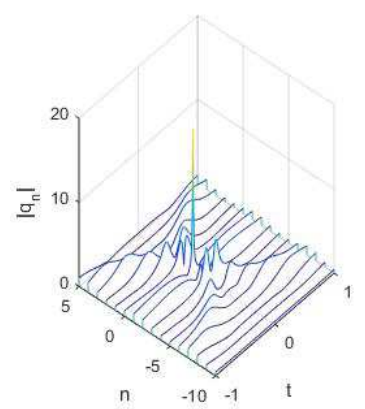

(a)

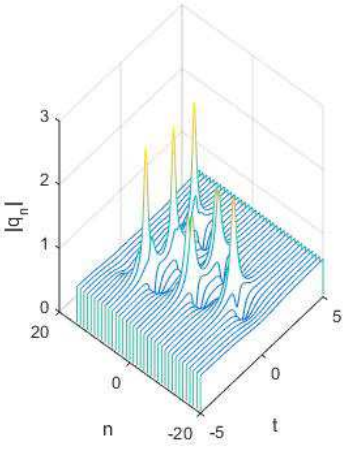

(b)

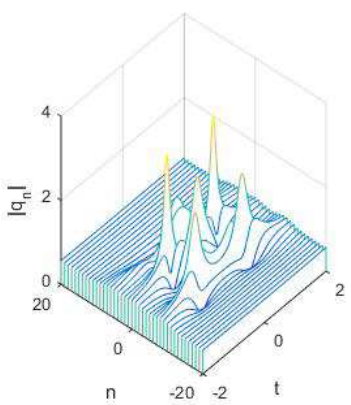

(c)

Figure 6: Third-order discrete RW solutions with $h=\gamma=1$ (a) strong interaction $d_{1,2}=f_{1,2}=0$, $n_{0}=0$; (b) a triangular pattern with $d_{1}=10, d_{2}=0, f_{1,2}=0, n_{0}=-1 / 2 ;$ (c) a pentagon pattern with $d_{1}=0, d_{2}=10, f_{1,2}=0, n_{0}=-1 / 3$.

with

$$
\Delta^{[3]}=\left|\begin{array}{cccccccc}
\phi_{1}[-4,0] & \phi_{1}[-4,1] & \phi_{1}[-4,2] & \phi_{1}[-4,3] & \psi_{1}[4,0]^{*} & \psi_{1}[4,1]^{*} & \psi_{1}[4,2]^{*} & \psi_{1}[4,3]^{*} \\
\psi_{1}[-4,0] & \psi_{1}[-4,1] & \psi_{1}[-4,2] & \psi_{1}[-4,3] & -\phi_{1}[4,0]^{*} & -\phi_{1}[4,1]^{*} & -\phi_{1}[4,2]^{*} & -\phi_{1}[4,3]^{*} \\
\phi_{1}[-2,0] & \phi_{1}[-2,1] & \phi_{1}[-2,2] & \phi_{1}[-2,3] & \psi_{1}[2,0]^{*} & \psi_{1}[2,1]^{*} & \psi_{1}[2,2]^{*} & \psi_{1}[2,3]^{*} \\
\psi_{1}[-2,0] & \psi_{1}[-2,1] & \psi_{1}[-2,2] & \psi_{1}[-2,3] & -\phi_{1}[2,0]^{*} & -\phi_{1}[2,1]^{*} & -\phi_{1}[2,2]^{*} & -\phi_{1}[2,3]^{*} \\
\phi_{1}[0,0] & \phi_{1}[0,1] & \phi_{1}[0,2] & \phi_{1}[0,3] & \psi_{1}[0,0]^{*} & \psi_{1}[0,1]^{*} & \psi_{1}[0,2]^{*} & \psi_{1}[0,3]^{*} \\
\psi_{1}[0,0] & \psi_{1}[0,1] & \psi_{1}[0,2] & \psi_{1}[0,3] & -\phi_{1}[0,0]^{*} & -\phi_{1}[0,1]^{*} & -\phi_{1}[0,2]^{*} & -\phi_{1}[0,3]^{*} \\
\phi_{1}[2,0] & \phi_{1}[2,1] & \phi_{1}[2,2] & \phi_{1}[2,3] & \psi_{1}[-2,0]^{*} & \psi_{1}[-2,1]^{*} & \psi_{1}[-2,2]^{*} & \psi_{1}[-2,3]^{*} \\
\psi_{1}[2,0] & \psi_{1}[2,1] & \psi_{1}[2,2] & \psi_{1}[2,3] & -\phi_{1}[-2,0]^{*} & -\phi_{1}[-2,1]^{*} & -\phi_{1}[-2,2]^{*} & -\phi_{1}[-2,3]^{*}
\end{array}\right|,
$$

and $\Delta_{1}^{[3]}$ and $\Delta_{2}^{[3]}$ change to $\Delta^{[3]}$ but the first row and second row in the $\Delta^{[3]}$ are replaced by $\left(\phi_{1}[4,0], \phi_{1}[4,1], \phi_{1}[4,2], \phi_{1}[4,3], \psi_{1}[-4,0]^{*}, \psi_{1}[-4,1]^{*}, \psi_{1}[-4,2]^{*}, \psi_{1}[-4,3]^{*}\right)$, respectively. The exact expression of three-order RW solution is so clumsy that we omit it here.

We just give its structural analysis corresponding to the four different parameters $\left(d_{1,2}, f_{1,2}\right)$.

- For the case $d_{1,2}=f_{1,2}=0$, the strong interaction is displayed in Figure 6(a).

The weak interaction happens when $d_{1} \neq 0$ or $d_{2} \neq 0, f_{1,2}=0$.

- For the case $d_{1}=10, d_{2}=f_{1,2}=0$, the third-order RWs split into six first-order RWs, which form a triangular pattern(see Fig. 6(b));

- For the case $d_{2}=10, d_{1}=f_{1,2}=0$, the third-order RWs also split into six first-order RWs, which array to a rotating pentagon pattern with a first-order RWs located at the center(see Fig. 6(c)).

Now we study the dynamical behaviors for the third-order RWs(18) by the numerical simulation. Here, we only consider the strong-interaction case(see Fig. 6(a)) and weak interaction case(see Fig. 


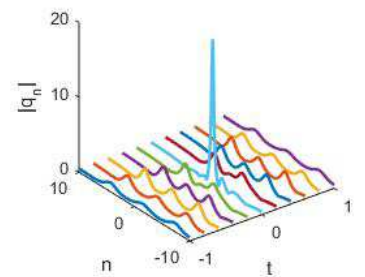

(a)

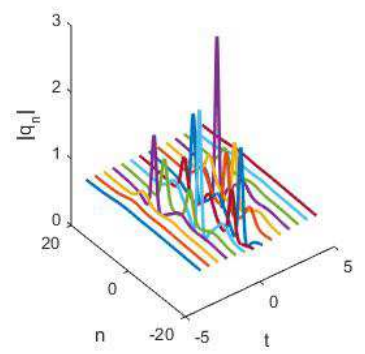

(d)

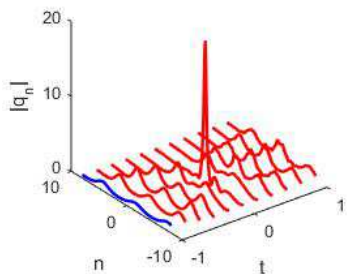

(b)

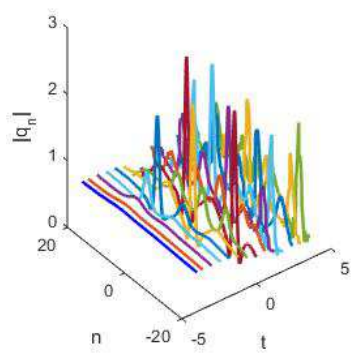

(e)

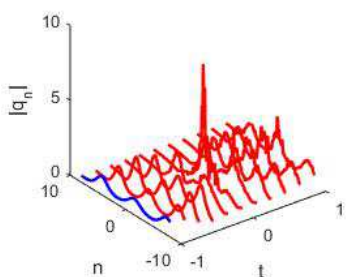

(c)

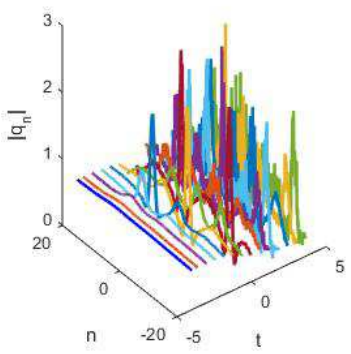

(f)

Figure 7: The third-order RW solutions(18). The exact solution (a) $d_{1,2}=f_{1,2}=0$ and (d) $d_{1}=10, d_{2}=$ $f_{1,2}=0$ (left); (b) and (e) simulated simulation using exact solutions(18) as the initial conditions (middle); (c) and (f) numerical simulations by adding random noise with amplitude $2 \%$ to the exact solutions(18) as initial conditions (right).

6(b)). Figures 7(a) and 7(b) show that the strong-interactions of third-order RWs nearly agree with the exact solution(18). If a small noise adds to the exact solution(18) in strong-interaction case, the wave propagation behaves well except a small bulge around the edges(see Fig. 7(c)). but the amplitude is obviously lower than the one's of the exact solution and numerical simulation case(see Figs. 7(a),7(b) and $7(\mathrm{c})$ ). On the other hand, no matter what we add a noise or not to the the initial condition, the the wave propagations of weak-interaction of third-order RWs display strong oscillations(see Fig.7(d)$7(f)$ ). We may infer that the dispersed energy of the third-order RWs is easier resulting in the disorder than the strong-interaction case.

\section{Modulation instability of continuous-wave states}

Many studies [24-28] have shown that the modulational instability(MI) associated with the growth of perturbations on a plane wave background can result in the RWs. We consider the continuous-wave 
solution of Eq.(2) $q_{0}(n, t)=c e^{i \phi t}$, where the real amplitude $c \neq 0$. We perturb this solution

$$
q_{n}(t)=\left(1+\frac{\varepsilon}{c} \hat{q}_{n}(t)\right) q_{0}(n, t),
$$

where $\varepsilon$ is an infinitesimal amplitude and $\hat{q}_{n}(t)$ is a perturbation solution. Substituting (19) into Eq.(2) yields a complex linearized equation

$$
\begin{aligned}
& i \hat{q}_{n, t}+\gamma\left(1+c^{2}\right)^{2} \hat{q}_{n-2}+\left(1+c^{2}+4 \gamma\left(c^{2}-1\right)\right) \hat{q}_{n-1}+\left(2 \gamma\left(c^{2}+1\right)\left(c^{2}-3\right)-2\right) \hat{q}_{n}+\gamma\left(c^{2}+1\right)^{2} \hat{q}_{n+2} \\
& +\left(c^{2}+1\right)\left(4 \gamma\left(c^{2}-1\right)+1\right) \hat{q}_{n+1}+2 c^{2} \gamma\left(1+c^{2}\right) \hat{q}_{n-1}^{*}+2 c^{2}(1-2 \gamma+4 c \gamma) \hat{q}_{n}^{*}+2 c^{2} \gamma\left(1+c^{2}\right) \hat{q}_{n+1}^{*}=0 .
\end{aligned}
$$

We consider the perturbation with real and imaginary parts $\hat{q}_{n}=q_{1, n}(t)+i q_{2, n}(t)$, which changes the the complex linearized equation can change into two real equations

$$
\begin{aligned}
& \gamma\left(1+c^{2}\right)^{2} q_{1, n-2}+\left(1+c^{2}\right)\left(1-4 \gamma+6 \gamma c^{2}\right) q_{1, n-1}+2\left(-1+c^{2}+\gamma\left(3-4 c^{2}+5 c^{4}\right)\right) q_{1, n} \\
& \left.+\left(1+c^{2}\right) \gamma\left(-4+6 c^{2}\right)\right) q_{1, n+1}+\left(1+c^{2}\right)^{2} q_{1, n+2}-q_{2, n, t}=0 \\
& \left(1+c^{2}\right)\left[\gamma\left(1+c^{2}\right) q_{2, n-2}+\left(1+2 \gamma\left(c^{2}-2\right)\right) q_{2, n-1}-2\left(1+3 \gamma\left(c^{2}-1\right)\right) q_{2, n}\right. \\
& \left.+\left(1+2 \gamma\left(c^{2}-2\right)\right) q_{2, n+1}+\gamma\left(1+c^{2}\right) q_{2, n+2}\right]+q_{1, n, t}=0 .
\end{aligned}
$$

Assume that real equation (21) exists the following complex solution

$$
\left(q_{1, n}(t), q_{2, n}(t)\right)=\left(q_{1, n}^{(0)}, q_{2, n}^{(0)}\right) e^{g t+i k n},
$$

where $g$ is the MI gain, $k$ is an arbitrary real wavenumber, and $q_{1, n}^{(0)}, q_{2, n}^{(0)}$ are constant amplitudes of the perturbation eigenmode. Substituting(22) into Eq.(21) yields the MI dispersion equation in the form of the determinant

$$
\left|\begin{array}{cc}
-g & 2\left(c^{2}-1+\left(c^{2}+1\right) \cos k\right)\left(1+2 \gamma\left(2 c^{2}-1\right)+2 \gamma\left(c^{2}+1\right) \cos k\right) \\
-4\left(c^{2}+1\right)\left(1+2 \gamma\left(c^{2}-1\right)+2 \gamma\left(c^{2}+1\right) \cos k\right) \sin ^{2} \frac{k}{2} & g
\end{array}\right|=0,
$$

which gives an explicit dispersion relation

$$
g^{2}=8\left(1+c^{2}\right)\left(c^{2}-1+\left(c^{2}+1\right) \cos k\right)\left(1+2 \gamma\left(2 c^{2}-1\right)+2 \gamma\left(1+c^{2}\right) \cos k\right)^{2} \sin ^{2} \frac{k}{2} .
$$

We point out here the MI takes place when expression(24) is positive. The MI condition $g^{2}>0$ holds as

$$
\cos k>\frac{1-c^{2}}{1+c^{2}}, \cos k \neq-\frac{1+2 \gamma\left(2 c^{2}-1\right)}{2 \gamma\left(1+c^{2}\right)}, \text { and } k \neq 2 m \pi, m \in Z
$$

Figure 8 shows that the growth rate $g(k)$ becomes larger and larger as the amplitude $c$ increases, meanwhile, for the fixed amplitude $c=2, g_{\max }(k)$ also gets large with the increase of parameter $\gamma$. 


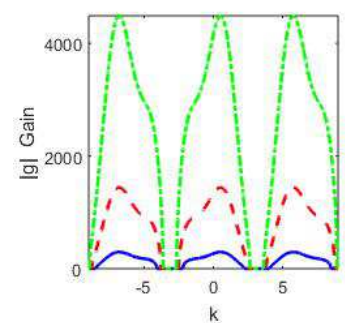

(a)

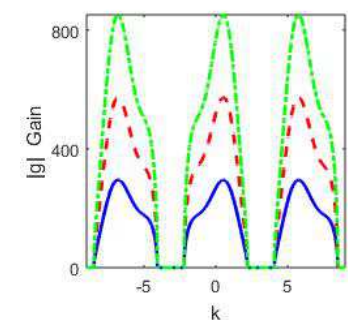

(b)

Figure 8: Gain spectra of the MI for different CW amplitude $c$ and higher order parameter $\gamma$. (a)Different amplitude with $c=2$ (blue solid), $c=3$ (red dashed), and $c=4$ (green dotted-dashed); (b)Different parameter with $\gamma=1$ (blue solid), $\gamma=2$ (red dashed), and $\gamma=3$ (green dotted-dashed).

\section{Conclusions}

In this paper, we have studied a higher-order integrable discrete NLS equation by the generalized discrete DT. The discrete multi-rogue wave solutions are given by determinants. We have analytically studied the dynamical behaviors of discrete RW solutions, which exhibit abundant patterns including on-site, inter-site, the rotating triangles and pentagons. Comparing with the discrete NLS equation, the first-order RWs of integrable higher-order discrete NLS equation have the same peaks with the same plane-wave amplitude but different center points. It is also shown that the nonlinear term parameter $\gamma$ has no effect on the amplitudes of the RWs but it can change the waveform. The time evolutions of the RW solutions are studied by means of numerical simulation, which reveals that strong-interaction RWs are stabler than the weak-interaction case. At last, the modulation instability condition of the background wave solutions are given.

Funding: This work of has been supported by the National Natural Science Foundations of China(grant number 12001361 and 11701510).

\section{Compliance with ethical standards}

Conflict of interest: The authors declare that they have no conflict of interest.

\section{References}

[1] Solli, D.R., Ropers,C., Koonath, P., Jalali, B.: Optical rogue waves. Nature 450, 06402 (2007) 
[2] Chabchoub, A., Hoffmann, N.P., Akhmediev, N.: Rogue wave observation in a water wave tank. Phys. Rev. Lett. 106, 204502 (2011)

[3] Yan, Z.Y.: Vector financial rogue waves. Phys. Lett. A 3754274 (2011)

[4] Guo, B.L., Ling, L.M., Liu, Q.P.: Nonlinear Schrödinger equation: generalized Darboux transformation and rogue wave solutions. Phys. Rev. E 85026607 (2012)

[5] Akhmediev, N., Ankiewicz, A., Taki, M.: Waves that appear from nowhere and disappear without a trace. Phys. Lett. A 373675 (2009)

[6] Li C.Z., He, J.S., Porsezian, K.: Rogue waves of the Hirota and the Maxwell-Bloch equations. Phys. Rev. E 87012913 (2013)

[7] Qiu, D.Q., He, J.S., Zhang, Y.S., Porsezian, K.: The Darboux transformation of the Kundu-Eckhaus equation. Proc. R. Soc. A 471, 20150236 (2015)

[8] Wang M., Chen Y.:Dynamic behaviors of mixed localized solutions for the three-component coupled FokasCLenells system. Nonlinear Dyn. 98 1781-1794 (2019)

[9] Yang, J., Zhu, Z.N.: Higher-order rogue wave solutions to a spatial discrete Hirota equation. Chin. Phys. Lett. 35090201 (2018)

[10] Bandelow, U., Akhmediev, N.: Persistence of rogue waves in extended nonlinear Schrödinger equations: Integrable Sasa-Satsuma case. Phys. Lett. A 3761558 (2012)

[11] Wen, X.Y., Yan, Z.Y., Boris A, Malomed: Higher-order vector discrete rogue-wave states in the coupled Ablowitz-Ladik equations: Exact solutions and stability. Chaos 26123110 (2016)

[12] Wen, X.Y., Yan, Z.Y.: Modulational instability and dynamics of multi-rogue wave solutions for the discrete Ablowitz-Ladik equation. J. Math. Phys. 59073511 (2018)

[13] Ohta, Y., Yang, J.K.: General rogue waves in the focusing and defocusing Ablowitz-Ladik equations. J. Phys. A: Math. Theor. 47255201 (2014)

[14] Lakshmanan, M., Porsezian, K., Daniel, M.: Effect of discreteness on the continuum limit of the Heisenberg spin chain, Phys. Lett. A 1339 (1988).

[15] Daniel, M., Latha, M.M.: Soliton in discrete and continuum alpha helical proteins with higher-order excitations. Physica A 240526 (1997)

[16] Daniel, M., Latha M.M.: A generalized Davydov soliton model for energy transfer in alpha helical proteins, Physica A 298 351-370 (2001)

[17] Porsezian, K., Daniel, M., Lakshmanan M.: On the integrability aspects of the onedimensional classical continuum isotropic biquadratic Heisenberg spin chain, J. Math. Phys. 33 1807-1816 (1992) 
[18] Saravana, Veni.S., Latha, M.M.: Nonlinear excitations in a disordered alpha-helical protein chain, Physica A 407 76-85 (2014)

[19] Kano, T.: Normal form of nonlinear Schrödinger equation, J. Phys. Soc. Jpn. 584322 (1989).

[20] Chowdury, A., Kedziora, D.J., Ankiewicz, A., Akhmediev, N.: Soliton solutions of an integrable nonlinear Schrödinger equation with quintic terms. Phys. Rev. E 90032922 (2014)

[21] Yang Y.Q., Yan Z.Y., and Malomed Boris A.: Rogue waves, rational solitons, and modulational instability in an integrable fifth-order nonlinear Schrödinger equation, Chaos 251031122015

[22] Chen S.Y., Yan Z.Y.: The higher-order nonlinear Schrödinger equation with non-zero boundary conditions: Robust inverse scattering transform, breathers,and rogons, Phys. Lett. A 383125906 (2019)

[23] Pickering, A., Zhao, H.Q., Zhu, Z.N.: On the continuum limit for a semidiscrete Hirota equation. 472 20160628 (2016)

[24] Ling, L.M., Zhao, L.C., Yang, Z.Y., Guo, B.L.: Generation mechanisms of fundamental rogue wave spatial-temporal structure. Phys. Rev. E 96022211 ( 2017)

[25] Dudley, J.M., Genty, G., Dias F., Kibler, B., Akhmediev N.: Modulation instability, Akhmediev Breathers and continuous wave supercontinuum generation. Opt. Express 1721497 (2009)

[26] Baronio, F., Conforti, M., Degasperis A., Lombardo, S., Onorato, M., Wabnitz, S.: Vector rogue waves and baseband modulation instability in the defocusing regime. Phys. Rev. Lett. 113034101 (2014)

[27] Baronio, F., Chen, S., Grelu, P., Wabnitz, S., Conforti, M.: Baseband modulation instability as the origin of rogue waves. Phys. Rev. A 91033804 (2015)

[28] Wang, X., Wei, J., Wang, L., Zhang, J.X.: Baseband modulation instebility, rogue waves and state transitions in a deformed Fokas-Lenells equation, Nonlinear Dyn. 97 343-353 (2019) 
Figures

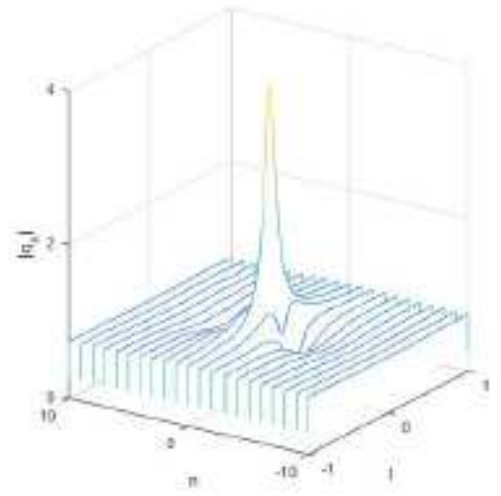

(a)

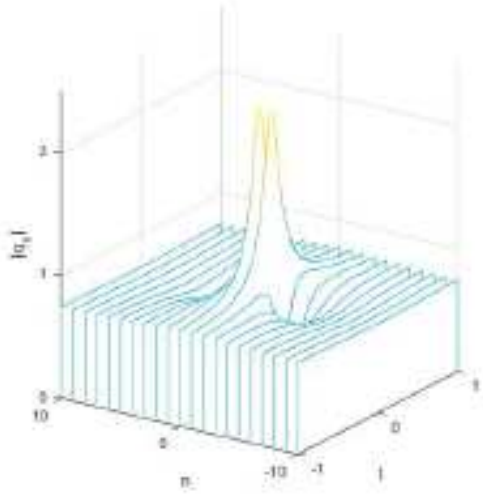

(b)

\section{Figure 1}

See the Manuscript Files section for the complete figure caption.

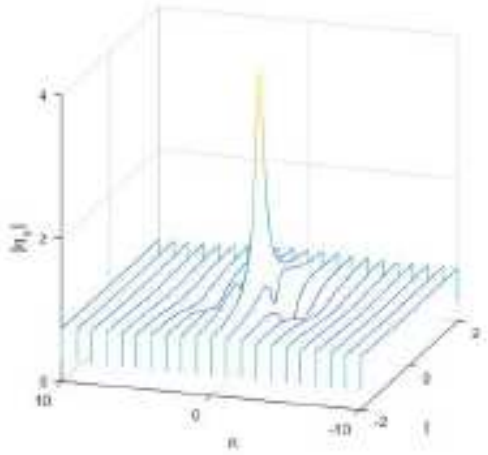

(a)

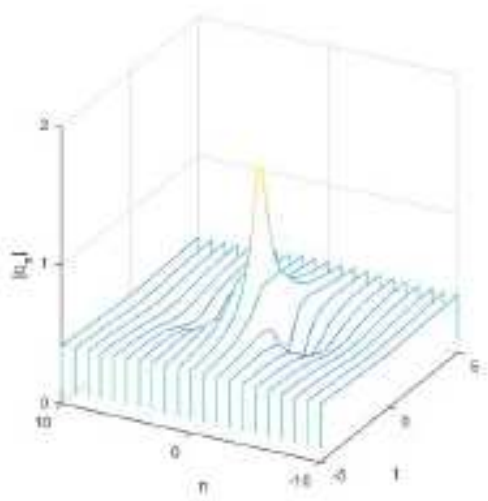

(d)

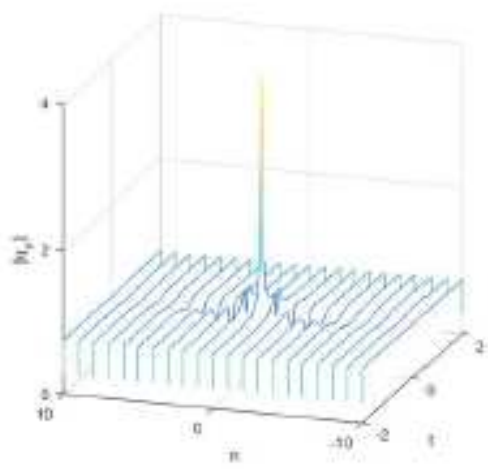

(b)

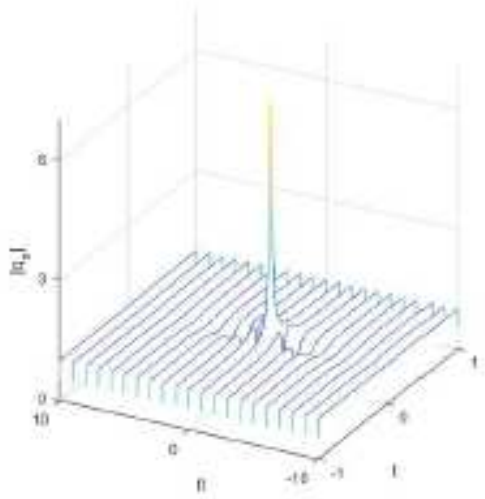

(e)

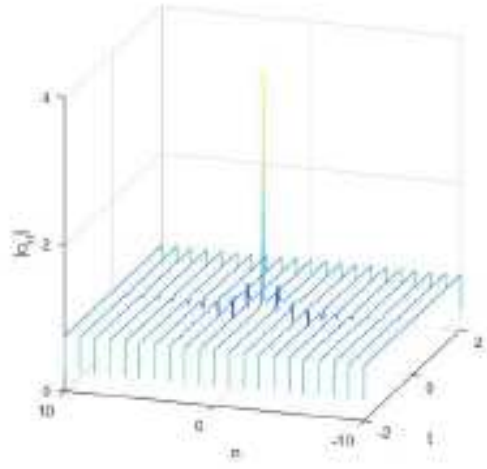

(c)

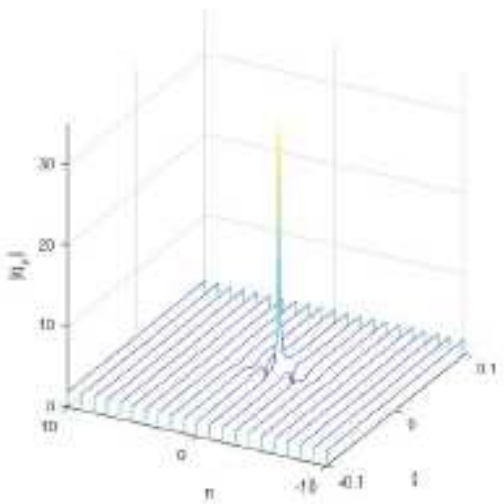

(f) 
Figure 2

See the Manuscript Files section for the complete figure caption.

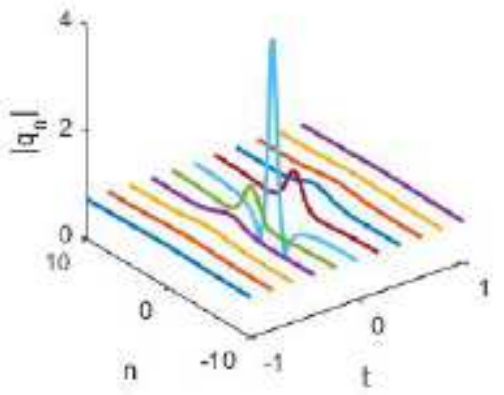

(a)

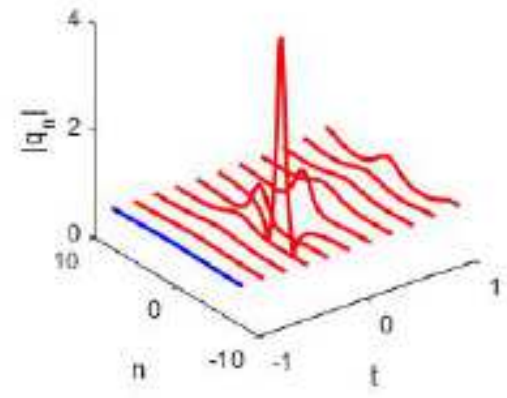

(b)

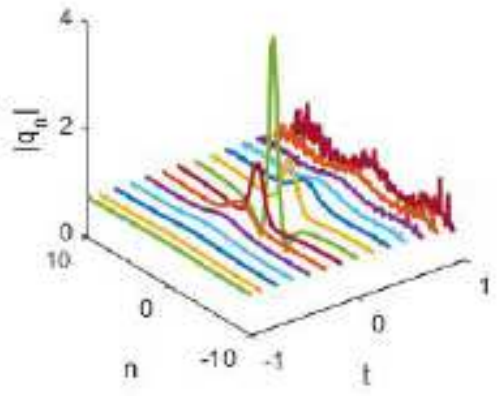

(c)

\section{Figure 3}

See the Manuscript Files section for the complete figure caption.

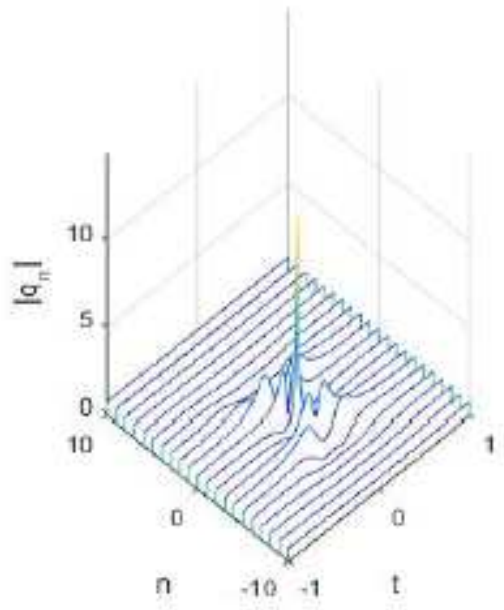

(a)

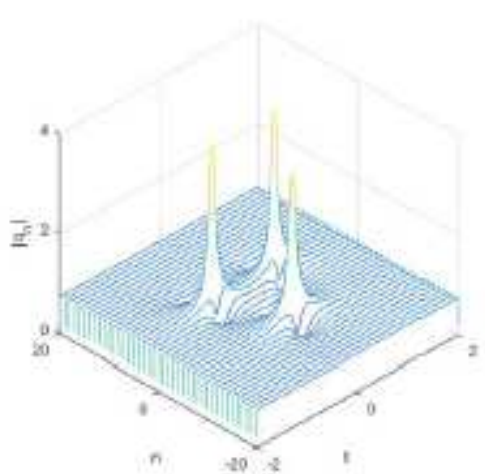

(b)

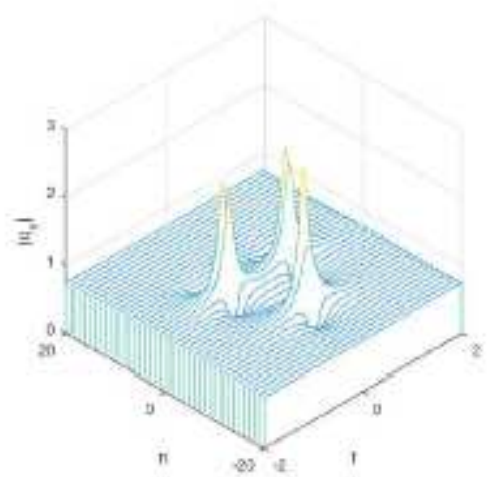

(c)

\section{Figure 4}

See the Manuscript Files section for the complete figure caption. 


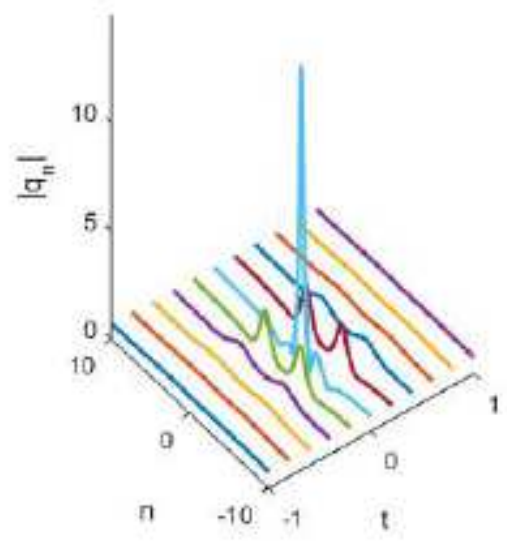

(a)

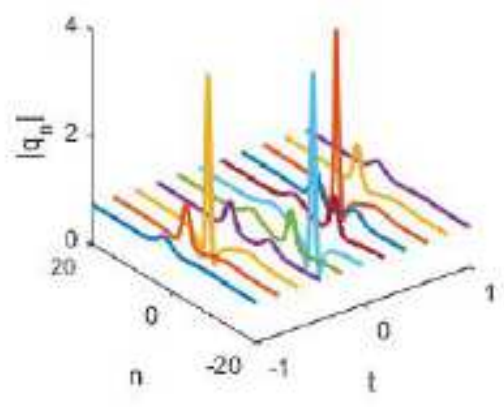

(d)

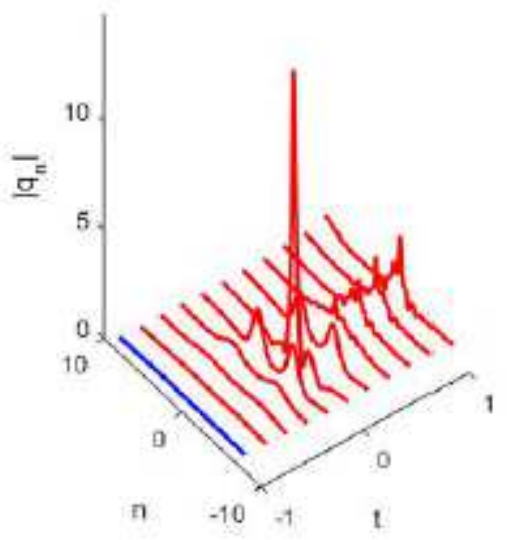

(b)

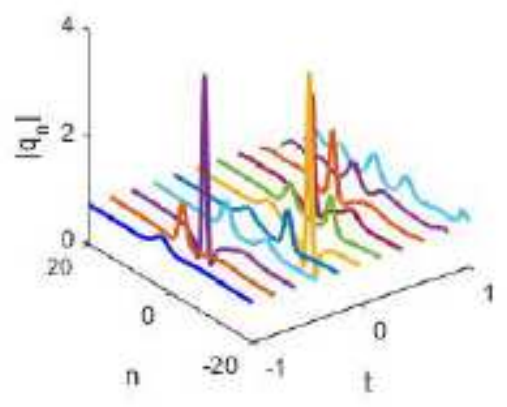

(e)

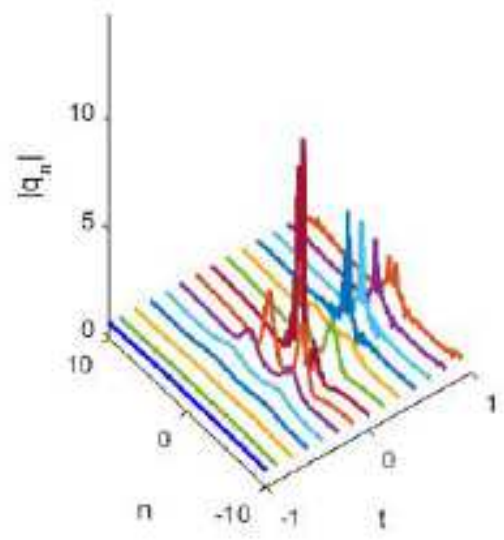

(c)

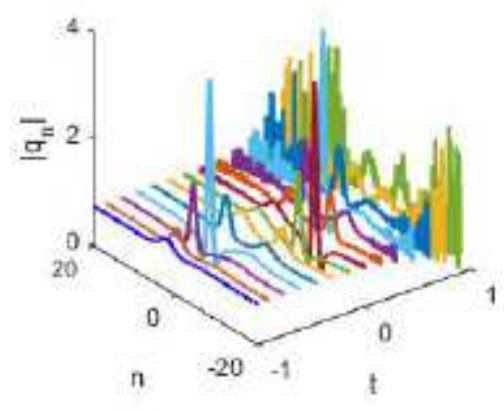

(f)

Figure 5

See the Manuscript Files section for the complete figure caption. 


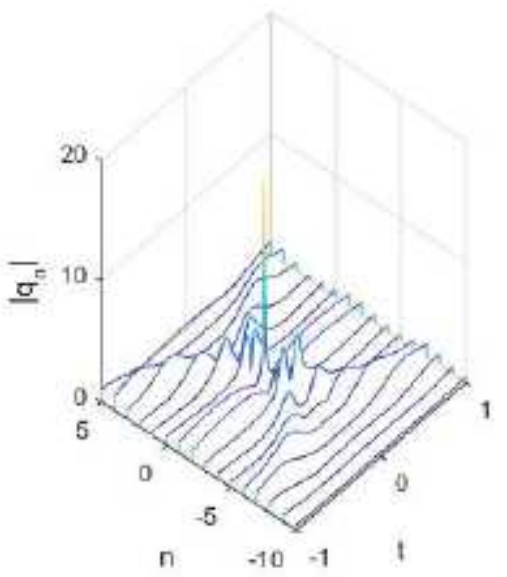

(a)

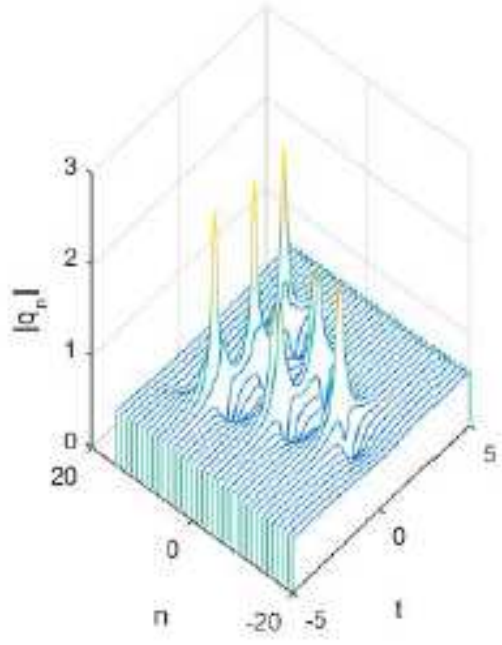

(b)

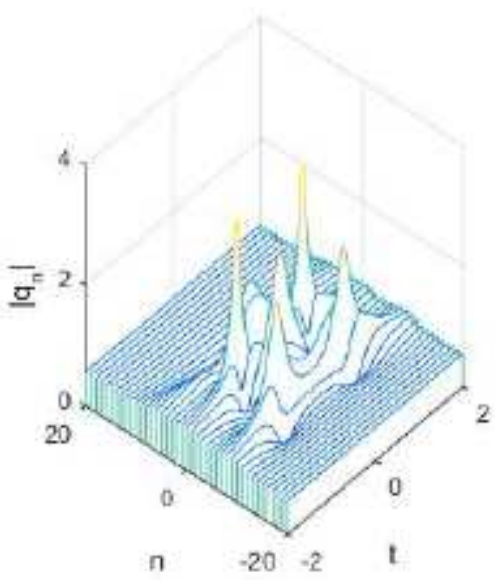

(c)

\section{Figure 6}

See the Manuscript Files section for the complete figure caption. 


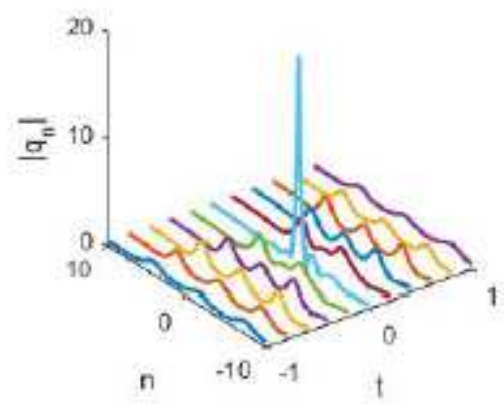

(a)

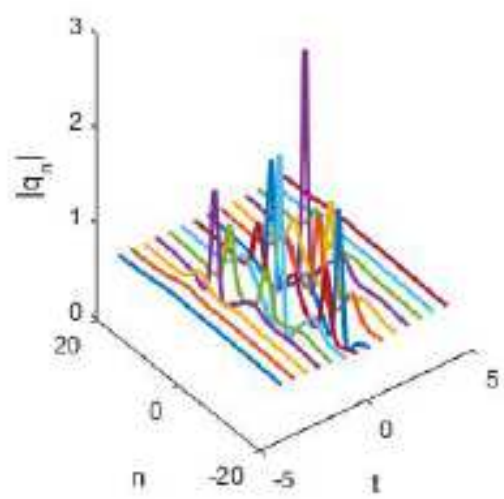

(d)

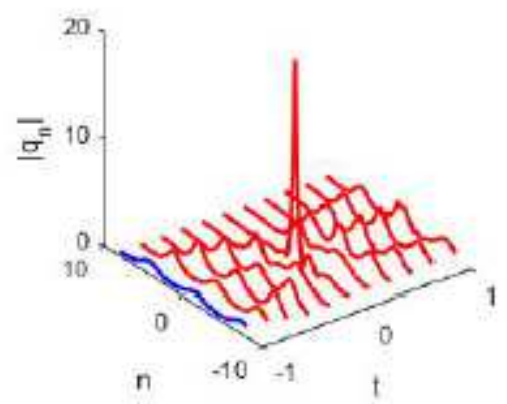

(b)

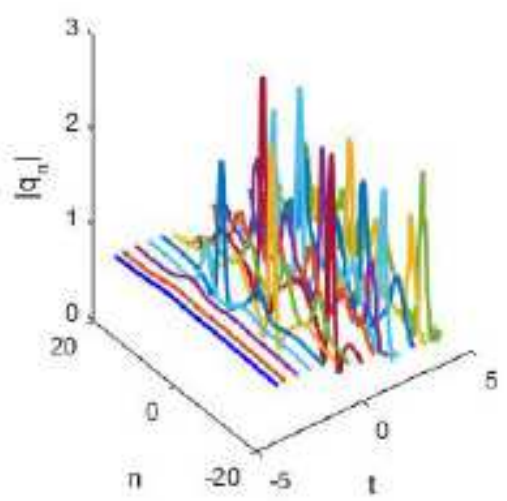

(e)

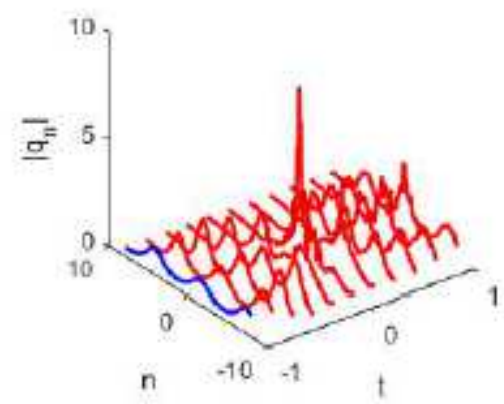

(c)

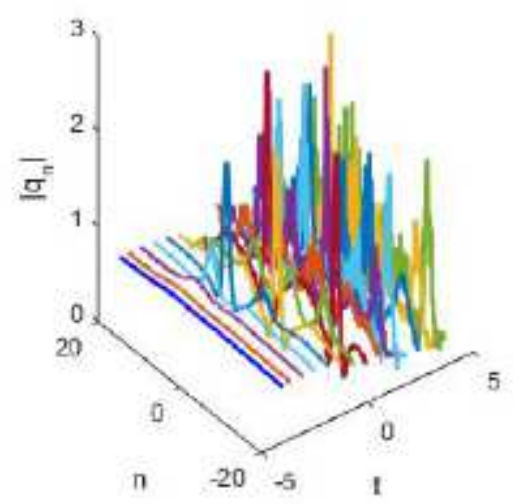

(f)

Figure 7

See the Manuscript Files section for the complete figure caption.

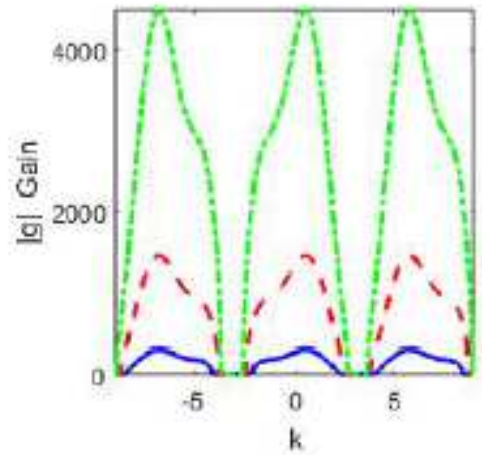

(a)

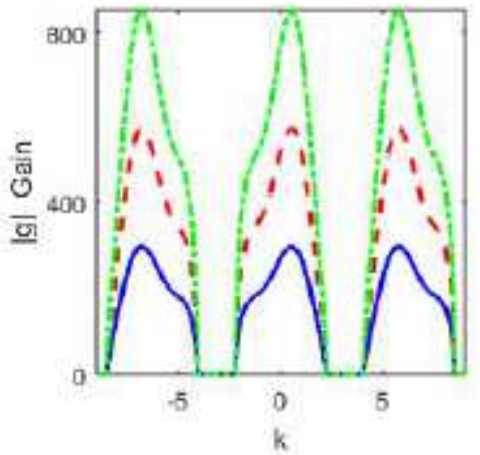

(b)

Figure 8

See the Manuscript Files section for the complete figure caption. 